\title{
ON COMPACTNESS AND LOEB MEASURES
}

\author{
J. M. ALDAZ
}

(Communicated by Andreas R. Blass)

\begin{abstract}
The purpose of this note is to show that neither a Loeb measure nor the image of a Loeb measure have to be compact, thus answering in the negative two questions of $D$. Ross.
\end{abstract}

D. Ross asked in a recent paper whether Loeb measures are always compact [D, Problem 1] and whether the image of every Loeb measure is compact [D, Problem 2]. Using 0-1 measures I will show that both questions have negative answers. A typical application of the Loeb measure construction goes as follows: Given a standard measure, find a Loeb measure which represents it and then work within a nonstandard model instead of using the original measure. This allows one to obtain standard results by utilizing the special properties of nonstandard models. It is therefore a central question in nonstandard measure theory to determine which measures can be represented by Loeb measures. Both of Ross's problems address this issue. Early on Anderson [A] proved that Radon measures have Loeb preimages, a result extended by Ross [D] to arbitrary compact measures. A positive answer to the first problem would have allowed (by Ross's result) the use of Loeb measures to represent other Loeb measures, making it possible to use even higher saturation conditions than those of the original model. The second example given below shows that the converse of Ross's result does not hold; that is, a "pushed down" Loeb measure need not be compact.

Recall that a (finite) measure is compact if it is inner regular with respect to a compact family. A family of sets is said to be compact if every subfamily with the finite intersection property (finite intersections are nonempty) has nonempty intersection. Prime examples of compact families are the compact subsets of a Hausdorff topological space and the closed subsets of a (not necessarily Hausdorff) compact space. Thus compact measures constitute a generalization of Radon measures. The following lemma will be used to answer both of Ross's questions.

1. Lemma. Let $(X, \mathscr{A}, \mu)$ be a measure space such that all points are measurable. If $\mu$ is a 0-1 measure which vanishes on points, then $\mu$ is not compact. Proof. Let $\mu$ be inner regular with respect to the family $\mathscr{K}$. It will be shown that $\mathscr{K}$ is not compact. For each $x \in X$, select $K_{x} \in \mathscr{K}$ with $K_{x} \subset X \backslash\{x\}$

Received by the editors December 30, 1992 and, in revised form, April 14, 1993.

1991 Mathematics Subject Classification. Primary 28E05; Secondary 03H05, 28A12. 
and $\mu\left(K_{x}\right)=1$. Now $\left\{K_{x}: x \in X\right\}$ has the finite intersection property, since finite intersections have measure one. But $\bigcap_{x \in X} K_{x} \subset \bigcap_{x \in X} X \backslash\{x\}=\varnothing$.

Nonstandard models will be assumed to be $\kappa$-saturated, with $\kappa$ "large enough". Take $\mu$ to be a standard $0-1$ measure which vanishes on points. For instance, let $X$ be the first uncountable ordinal, let $\mathscr{A}$ be the $\sigma$-algebra of countable and cocountable subsets of $X$, and let $\mu$ be defined by $\mu(A)=0$ if $A$ is countable or $\mu(A)=1$ if $A$ is cocountable. By the lemma, $\left({ }^{*} X,{ }^{*} \mathscr{A}, L\left({ }^{*} \mu\right)\right)$ is not a compact measure space, since $L\left({ }^{*} \mu\right)$ is $0-1$ and vanishes on points. This answers Ross's first question.

In order to solve the second problem, I will show first that the image of a compact measure need not be compact. Regarding images of Loeb measures, Render has found a positive result in the case where the internal measure is $0-1$ $[R$, Theorem 4.6]. Let $c$ denote the cardinality of the continuum. It will be useful to recall some facts about the classical Vitali's construction of a nonmeasurable subset of $[0,1)$ (with respect to Lebesgue measure). The operation to be used on this group is addition $\bmod (1)$, and it shall be denoted simply by + . Now $[0,1)$ modulo the rationals is partitioned into $c$-many cosets (since each coset is countable). List the cosets $\{V \alpha: \alpha<c\}$, and for each $\alpha$ select $x_{\alpha} \in V \alpha$. Then the Vitali set $V=\bigcup_{\alpha<c}\left\{x_{c}\right\}$ is not measurable. Let $\left\{r_{n}: n \in \mathbb{N}\right\}$ be a listing of the rationals in $[0,1)$. Since $\left\{V+r_{n}: n \in \mathbb{N}\right\}$ is a partition of $[0,1)$, it follows by translation invariance that the inner measure of $V$ is zero. It is well known, however, that this procedure does not fully specify its outer measure. If the choices are made in a suitable manner, the outer measure of $V$ can be taken to be one. This is the content of the next lemma.

2. Lemma. The Vitali set $V$ can be chosen so that its outer measure is one.

Proof. Let $\{C \alpha: \alpha<c\}$ be the collection of closed subsets of $[0,1)$ with positive Lebesgue measure. The set $C_{0}$ has nonempty intersection with some coset $V \alpha_{0}$. Pick $x_{0} \in C_{0} \cap V \alpha_{0}$. Assume $x_{\beta}$ has already been chosen for every $\beta<\gamma$. Since each coset is countable and the union of less than $c$ countable sets has cardinality less than $c$, it follows that $C_{\gamma} \backslash \bigcup_{\beta<\gamma} V \alpha_{\beta}$ is not empty. Hence, there exists an $\alpha_{\gamma} \neq \alpha_{\beta}$ for all $\beta<\gamma$ such that $C_{\gamma} \cap V \alpha_{\gamma}$ is not empty. Pick $x_{\gamma} \in C_{\gamma} \cap V \alpha_{\gamma}$. Finally, select one point from each coset not represented in $\bigcup_{\gamma<c}\left\{x_{\alpha_{\gamma}}\right\}$ (if there is any) and add these points to $\bigcup_{\gamma<c}\left\{x_{\alpha_{\gamma}}\right\}$. In this way we obtain a set $V$ with inner measure zero and outer measure one, which contains exactly one representative from each coset.

3. Theorem. The image of a compact measure under an arbitrary map need not be compact.

Proof. Let $\lambda$ be the Lebesgue measure on $[0,1)$, and let $V$ be a Vitali set with $\lambda^{*}(V)=1$. The idea is to find a function $f:[0,1) \rightarrow V$ such that $\lambda \circ f^{-1}$ is a $0-1$ measure vanishing on points. Let $f_{n}: V+r_{n} \rightarrow V$ be the natural bijection $f_{n}(x)=x-r_{n}$. Set $f=\bigcup f_{n}$. Since the functions $f_{n}$ have disjoint domains, it follows that $f$ is a function. Now every point of $V$ has a countable preimage, so $\lambda \circ f^{-1}(\{x\})=0$ for all $x \in V$. By the definition of $f$, if $A \subset V$ and $x \in f^{-1}(A)$, then $x+r_{n} \in f^{-1}(A)$ for all rationals $r_{n}$. Suppose $A$ is $\left(\lambda \circ f^{-1}\right)$-measurable. Given $n \in \mathbb{N}$, the measure of the intersection between $(0,1 / n)$ and $f^{-1}(A)$ is simply the product of their measures, and the same is true for every rational translate $\bmod (1)$ of $(0,1 / n)$. Form the outer 
regularity of Lebesgue measure, it follows that given any measurable set $E$, $\lambda\left[E \cap f^{-1}(A)\right]=\lambda(E) \lambda\left[f^{-1}(A)\right]$. Setting $E=f^{-1}(A)$ one sees that $\lambda\left[f^{-1}(A)\right]$ is either 0 or 1 , whence $\lambda \circ f^{-1}$ is not compact (Lemma 1).

4. Corollary. The image of a Loeb measure need not be compact.

Proof. Consider $L\left({ }^{*} \lambda\right)$ on ${ }^{*}[0,1)$. Let st denote the standard part map, and let $f$ be the function defined in the proof of Theorem 3. Then the measure $L\left({ }^{*} \lambda\right)\left(\mathrm{st}^{-1}\left[f^{-1}(\cdot)\right]\right)$ is not compact.

It also follows that the image of an $\alpha$-compact measure need not be $\alpha$ compact, since Loeb measures are $\alpha$-compact for every $\alpha$ below the saturation of the model (see [D] for the definition of $\alpha$-compactness).

Note that the last example can easily be modified so that $g:{ }^{*}[0,1) \rightarrow[0,1)$ is onto, $g\left({ }^{*} x\right)=x$, and $L\left({ }^{*} \lambda\right) \circ g^{-1}$ is not compact. First extend $f \circ$ st to ${ }^{*}[0,1)$ by mapping ${ }^{*}[0,1) \backslash n s\left({ }^{*}[0,1)\right)$ onto $[0,1) \backslash V$, and then change (when needed) its values at the points ${ }^{*} x$, mapping ${ }^{*} x$ to $x$. Since both sets ${ }^{*}[0,1) \backslash n s\left({ }^{*}[0,1)\right)$ and $\left\{{ }^{*} x: x \in[0,1)\right\}$ have $L\left({ }^{*} \lambda\right)$-measure zero, the arguments given before remain valid.

The preceding examples raise some new open problems (most of them suggested by the referee), namely: (1) To characterize the measures that result from "pushing down" Loeb measures. (2) To determine which types of functions generate compact images from Loeb measures. (3) Can a nonatomic Loeb measure be compact? Two interesting (and related) special cases of question (3) are: (4) Is the "usual" Loeb probability measure (generated by an internal hyperfinite counting measure on $\left.{ }^{*}[0,1]\right)$ compact? (5) Let $\lambda$ denote the Lebesgue measure on $[0,1]$; is $\left.L{ }^{*} \lambda\right)$ compact? With regard to the last two questions, I believe that the answer is no, at least for models with saturation larger than the cardinality of the continuum.

\section{ACKNOWLEDGMENT}

The author is indebted to the referee for several useful comments.

\section{REFERENCES}

[A] R. Anderson, Star-finite representations of measure spaces, Trans. Amer. Math. Soc. 271 (1981), 667-687.

[D] D. Ross, Compact measures have Loeb preimages, Proc. Amer. Math. Soc. 115 (1992), 356-370.

[R] H. Render, Pushing down Loeb measures, Math. Scand. 72 (1993), 61-84.

Departamento de Matematicas, Universidad Autonoma de Madrid, Spain

E-mail address: aldaz@ccuam3.sdi.vam.es 